Preprint typeset in JHEP style - HYPER VERSION

\title{
Poisson-Lie T-dual sigma models on supermanifolds
}

\author{
A. Eghbali, A. Rezaei-Aghdam \\ Department of Physics, Faculty of science, Azarbaijan University of Tarbiat Moallem, \\ 53714-161, Tabriz, Iran \\ E-mail: a.eghbali@azaruniv.edu, \\ Corresponding Author: rezaei-a@azaruniv.edu
}

\begin{abstract}
We investigate Poisson-Lie symmetry for T-dual sigma models on supermanifolds in general and on Lie supergroups in particular. We show that the integrability condition on this super Poisson-Lie symmetry is equivalent to the super Jacobi identities of the Lie super-bialgebras. As examples we consider models related to four dimensional Lie super-bialgebras $\left(\left(2 A_{1,1}+2 A\right)^{1}, D_{p=\frac{1}{2}}^{10}\right)$ and $\left(\left(2 A_{1,1}+2 A\right)^{1}, I\right)$. Then generally it is shown that for Abelian case $(\mathrm{g}, I)$ the super Poisson-Lie T-duality transforms the role of fermionic (bosonic) fields in the model to bosonic (fermionic) fields on the dual model and vice versa.
\end{abstract}

Keywords: Sigma Models, String Duality. 


\section{Contents}

1. Introduction 1

2. Super Poisson-Lie symmetry in sigma models on supermanifolds 2

3. Super Poisson-Lie T-dual sigma models on supergroups 3

4. Examples 5

4.1 Case A

4.2 Case B 8

5. Super non-Abelian duality 10

6. Conclusion 11

\section{Introduction}

Field theories with supermanifold as target space have recently received considerable attention, because of their interesting applications in both string theory and condensed matter physics. $W Z N W$ models on supergroups are related to local logarithmic conformal field theories [1, 2, 3] . Moreover, sigma model on Lie supergroups provides the building blocks of string theory in $A d S$ backgrounds; for example, superstring theory on $A d S_{3} \times S^{3}$ is related to a sigma model on $P S U(1,1 \mid 2)$ [4, 5, 6]. Furthermore, the study of two-dimensional topological sigma model on supermanifolds gives a better understanding of mirror symmetry and its relation to $T$-duality [0, \&]. $T$-duality is the most important symmetries of string theory [9]. On the other hand Poisson-Lie $T$-duality, a generalization of $T$-duality, does not require existence of isometry in the original target manifold (as in usual $T$-duality) [10, 11, 12, 13]; the integrability of the Noether's currents associated with the action of group $G$ on the target manifold is enough to have this symmetry. In these models, the components of the Noether's currents play the role of flat connection, i.e. they satisfy Maurer-Cartan equations with group structure of $\tilde{G}$ (with the same dimension of $G$ ) [10, 11] so that $G$ and $\tilde{G}$ have Poisson-Lie structure and their Lie algebras form a Lie bialgebra [14. In [15] it is shown that Poisson-Lie $T$-duality in $(2,2)$ superconformal $W Z N W$ and Kazama-Suzuki models acts as a mirror symmetry. So the study of Poisson-Lie $T$-dual sigma models on supermanifolds and its relation to mirror symmetry is an interesting problem. However, to our knowledge Poisson-Lie $T$-dual sigma models on supermanifolds have not been studied until now. In this paper, we investigate this problem.

The paper is organized as follows. In section two we generalize the Poisson-Lie symmetry to sigma models on supermanifolds and show that the, integrability condition on this 
super Poisson-Lie symmetry gives the super Jacobi identities of Lie super-bialgebras [17. The super Poisson-Lie $T$-dual sigma models on supergroups are investigated in section three. As the fourth section, we give examples of four-dimensional Lie super-bialgebras $\left(\left(2 A_{1,1}+2 A\right)^{1}, D_{p=\frac{1}{2}}^{10}\right)$ 19] and $\left(\left(2 A_{1,1}+2 A\right)^{1}, I\right)$ 18 and for the second example we give canonical transformation that relate model and its dual one. Then in section five, we show that in general for the Abelian case $(\mathbf{g}, I)$ the super Poisson-Lie $T$-duality transforms the role of fermionic (bosonic) fields in the model to bosonic (fermionic) fields on the dual model and vice versa. Some remarks are discussed in as concluding section.

\section{Super Poisson-Lie symmetry in sigma models on supermanifolds}

Consider two dimentional sigma models on supermanifolds ${ }^{1} M$ as target space with background matrix ${ }_{\mu} \mathcal{E}_{\nu}(x)={ }_{\mu} G_{\nu}(x)+{ }_{\mu} B_{\nu}(x)$ as function of coordinates $x^{\mu 2}$

$$
S=\frac{1}{2} \int d \xi^{+} \wedge d \xi^{-} \partial_{+} x^{\mu}{ }_{\mu} \mathcal{E}_{\nu} \partial_{-} x^{\nu}=\frac{1}{2} \int d \xi^{+} \wedge d \xi^{-} L .
$$

Suppose that a supergroup $G$ acts from right on $M$ freely. Then with the use of left invariant supervector field ${ }_{i} v^{\mu(L, l)}$ (defined with left derivative)

$$
{ }_{i} v^{(L, l)}={ }_{i} v^{\mu(L, l)} \frac{\vec{\partial}}{\partial x^{\mu}} \quad,(i=1, \ldots, \operatorname{dim} G)
$$

corresponding to this action, one can compute variation of $S$ under transformation $x^{\mu} \longrightarrow$ $x^{\mu}+\epsilon^{i}\left(\xi^{+}, \xi^{-}\right)_{i} v^{\mu}$ as follows ${ }^{3}$ :

$$
\delta S=\frac{1}{2} \int d \xi^{+} \wedge d \xi^{-} \varepsilon^{i}(-1)^{\lambda+i \lambda} \partial_{+} x^{\lambda} \mathcal{L}_{i} v \mathcal{E}_{\lambda \nu} \partial_{-} x^{\nu}-\frac{1}{2} \int d \varepsilon^{i} \wedge \star_{i} J,
$$

where the Lie superderivative $\mathcal{L}_{i v} \mathcal{E}_{\lambda \nu}$ and Hodge star of Nother's current have the following forms respectively

$$
\begin{gathered}
\mathcal{L}_{i v} \mathcal{E}_{\mu \nu}=(-1)^{i \mu+\mu+\lambda} \overrightarrow{\partial_{\mu}}{ }_{i} v^{\lambda} \mathcal{E}_{\lambda \nu}+{ }_{i} v^{\lambda} \overrightarrow{\partial_{\lambda}} \mathcal{E}_{\mu \nu}+(-1)^{\mu \nu+\mu \lambda+i \nu+\lambda+\nu} \vec{\partial}_{\nu}{ }_{i} v^{\lambda} \mathcal{E}_{\mu \lambda}, \\
\star_{i} J=(-1)^{\mu+\lambda}{ }_{i} v^{\mu} \partial_{+} x^{\lambda} \mathcal{E}_{\lambda \mu} d \xi^{+}-(-1)^{\mu}{ }_{i} v^{\mu} \mathcal{E}_{\mu \nu} \partial_{-} x^{\nu} d \xi^{-} .
\end{gathered}
$$

By direct calculation, one can consider

$$
d \star_{i} J=\left[(-1)^{\lambda+i \lambda} \partial_{+} x^{\lambda} \mathcal{L}_{i v} \mathcal{E}_{\lambda \nu} \partial_{-} x^{\nu}+{ }_{i} v^{\mu} \text { (equations of motion) }\right] d \xi^{-} \wedge d \xi^{+},
$$

where the equations of motion have the following form

\footnotetext{
${ }^{1}$ Here we use the notation presented by DeWitt's in [16]. For example the transformation properties of upper and lower right indices to the left one are as follows:

$$
{ }^{i} T_{j l \ldots}^{k}=T_{j l \ldots}^{i k}, \quad{ }_{j} T_{l \ldots}^{i k}=(-1)^{j} T_{j l \ldots}^{i k},
$$

where indices on the exponent of $(-1)$ show the Grassmann degrees of variables.

${ }^{2}$ Here we consider bosonic worldsheet with light cone coordinates: $\xi^{ \pm}=\frac{1}{2}(\tau \pm \sigma)$

${ }^{3}$ From now on we will omit the superscripts $(L, l)$ on ${ }_{i} v^{\mu}$.
} 


$$
\begin{gathered}
-(-1)^{\lambda+\mu \lambda} \partial_{+} x^{\lambda} \overrightarrow{\partial_{\mu}} \mathcal{E}_{\lambda \nu} \partial_{-} x^{\nu}+(-1)^{\mu} \partial_{+} x^{\lambda} \overrightarrow{\partial_{\lambda}} \mathcal{E}_{\mu \nu} \partial_{-} x^{\nu}+(-1)^{\mu} \mathcal{E}_{\mu \nu} \partial_{+} \partial_{-} x^{\nu} \\
+(-1)^{\lambda+\mu} \partial_{-} \partial_{+} x^{\lambda} \mathcal{E}_{\lambda \mu}+(-1)^{\lambda+\mu} \partial_{+} x^{\lambda} \partial_{-} x^{\nu} \overrightarrow{\partial_{\nu}} \mathcal{E}_{\lambda \mu}=0 .
\end{gathered}
$$

Thus, on extremal surface we have $\delta S=0$ and

$$
d \star_{i} J=-\left[(-1)^{\lambda+i \lambda} \partial_{+} x^{\lambda} \mathcal{L}_{i v} \mathcal{E}_{\lambda \nu} \partial_{-} x^{\nu}\right] d \xi^{+} \wedge d \xi^{-} .
$$

If $\mathcal{L}_{i v} \mathcal{E}_{\lambda \nu}=0$, then $G$ is a superisometry group of $M$ and we have conserved currents. On the other hand, if $\star_{i} J$ on extremal surfaces satisfy Maurer-cartan equation [16]

$$
d \star_{i} J=-(-1)^{j k} \frac{1}{2} \tilde{f}_{i}^{j k} \star_{j} J \wedge \star_{k} J
$$

where, $\tilde{f}_{i}^{j k}$ are structure constants of Lie superalgebras $\tilde{\mathbf{g}}$ (with the same dimension of $\mathbf{g}$ (Lie superalgebra of $G$ )); then the super Poisson-Lie symmetry will be

$$
\mathcal{L}_{v_{i}}\left(\mathcal{E}_{\lambda \nu}\right)=(-1)^{\mu+\mu^{\prime}+\lambda \mu^{\prime}+j k+\mu k+\mu \mu^{\prime}+\nu \lambda+\mu^{\prime} \nu} \tilde{f}^{j k}{ }_{i j} v^{\mu}{ }_{k} v^{\mu \prime} \mathcal{E}_{\mu \nu} \mathcal{E}_{\lambda \mu^{\prime}},
$$

where this formula is a generalization of usual Poisson-Lie symmetry [10] to sigma models on supermanifolds.

Now, using integrability condition on Lie superderivative

$$
\mathcal{L}_{\left[i v,{ }_{j} v\right]}\left(\mathcal{E}_{\lambda \nu}\right)=\left[\mathcal{L}_{i v}, \mathcal{L}_{j} v\right] \mathcal{E}_{\lambda \nu}=\mathcal{L}_{i v} \mathcal{L}_{j v} \mathcal{E}_{\lambda \nu}-(-1)^{i j} \mathcal{L}_{j}{ }_{j} \mathcal{L}_{i v} \mathcal{E}_{\lambda \nu}
$$

and after some computations, we see that the structure constants of Lie superalgebras $\mathbf{g}$ and $\tilde{\mathbf{g}}$ must be satisfied in the following relations

$$
f^{k}{ }_{i j} \tilde{f}_{k}^{m l}=(-1)^{i l} f_{i k}^{m} \tilde{f}_{j}^{k l}+f_{i k}^{l} \tilde{f}_{j}^{m k}+f_{k j}^{m} \tilde{f}_{i}^{k l}+(-1)^{m j} f_{k j}^{l} \tilde{f}_{i}^{m k},
$$

where these are the mixed superJacobi identities of the Lie super-bialgebras $(\mathbf{g}, \tilde{\mathbf{g}})$ [18, 21]. In the same way, one can consider the dual sigma models with background matrix ${ }_{\mu} \tilde{\mathcal{E}}_{\nu}$; where the supergroup $\tilde{G}$ acts freely on $M$ and the roles of $\mathbf{g}$ and $\tilde{\mathbf{g}}$ are exchanged.

\section{Super Poisson-Lie T-dual sigma models on supergroups}

When the supergroup $G$ acts transitively and freely on $M$, the target can be identified with the supergroup $G$. In this case, in order to obtain T-dual sigma models, one can consider the equation of motion for the action on the Drinfeld superdouble $D$ [11]

$$
<\partial_{ \pm} l l^{-1}, \varepsilon^{\mp}>=0
$$

where $l\left(\xi^{+}, \xi^{-}\right)$is a map from world sheet to Drinfeld superdouble $D$ and $\langle.,$.$\rangle is the$ invariant bilinear form on the double and $\varepsilon^{\mp}$ are $n$ dimensional orthogonal super vector spaces such that $\varepsilon^{+}+\varepsilon^{-}$spans Lie superalgebra $\mathcal{D}=\mathbf{g} \bigoplus \tilde{\mathbf{g}}$. Now by using decomposition $l$ in the vicinity of the unit element of $D$ 17]

$$
l\left(\xi^{+}, \xi^{-}\right)=g\left(\xi^{+}, \xi^{-}\right) \tilde{h}\left(\xi^{+}, \xi^{-}\right), \quad(g \in G, \quad \tilde{h} \in \tilde{G})
$$


we obtain from $(3.1)$

$$
<g^{-1} \partial_{ \pm} g+\partial_{ \pm} \tilde{h} \tilde{h}^{-1}, g^{-1} \varepsilon^{\mp} g>=0 .
$$

On the other hand, for the super vector spaces $\varepsilon^{\mp}$ we have

$$
g^{-1} \varepsilon^{ \pm} g=\operatorname{Span}\left\{X_{i} \pm E_{i j}^{ \pm}(g) \tilde{X}^{j}\right\}
$$

where super matrices $E^{ \pm}$are supertranspose of each other $\left(E_{i j}^{-}=(-1)^{i j} E_{j i}^{+}\right),\left\{X_{i}\right\}$ and $\left\{\tilde{X}^{i}\right\}$ are bases of Lie superalgebras $\mathbf{g}$ and $\tilde{\mathbf{g}}$ such that

$$
\begin{gathered}
<X_{i}, X_{j}>=<\tilde{X}^{i}, \tilde{X}^{j}>=0 \\
<X_{i}, \tilde{X}^{j}>=\delta_{i}{ }^{j}=(-1)^{i j} \delta^{j}{ }_{i}=(-1)^{i j}<\tilde{X}^{j}, X_{i}>,
\end{gathered}
$$

and we have ${ }^{4}$

$$
E^{+}(g)=\left(a(g)+E^{+}(e) b(g)\right)^{-1} E^{+}(e) d(g),
$$

such that

$$
\begin{gathered}
g^{-1} X_{i} g=a(g)_{i}{ }_{k}{ }_{k} X=(-1)^{k} a(g)_{i}{ }^{k} X_{k}, \\
g^{-1} \tilde{X}^{j} g=b(g)^{j k}{ }_{k} X+d(g)^{j}{ }_{k} \tilde{X}^{k}=(-1)^{k} b(g)^{j k} X_{k}+d(g)^{j}{ }_{k} \tilde{X}^{k},
\end{gathered}
$$

Now by using (3.3)-(3.5) we have

$$
\begin{aligned}
& A_{+i}(g):=\left(\partial_{+} \tilde{h} \tilde{h}^{-1}\right)_{i}=(-1)^{l}\left(g^{-1} \partial_{+} g\right)^{l} E_{l i}^{+}(g), \\
& A_{-i}(g):=\left(\partial_{-} \tilde{h} \tilde{h}^{-1}\right)_{i}=-E_{i l}^{+}(g)\left(g^{-1} \partial_{-} g\right)^{l},
\end{aligned}
$$

where $A_{ \pm}$are right invariant one forms on $\tilde{G}$ and satisfy in the following flat connection relation

$$
\partial_{+} A_{-i}(g)-\partial_{-} A_{+i}(g)-(-1)^{j l} \tilde{f}_{i}^{j l} A_{-j}(g) A_{+l}(g)=0 .
$$

Indeed one can observe that the above equation results in the equations of motions and super Poisson-Lie symmetry of the following action

$$
S=\frac{1}{2} \int\left(g^{-1} \partial_{+} g\right)^{i}{ }_{i} E_{j}^{+}(g)\left(g^{-1} \partial_{-} g\right)^{j} d \xi^{+} d \xi^{-} .
$$

To see this it is convenient to use the following definition for the left invariant one forms with left derivative 17, 20

$$
\begin{aligned}
& \left(g^{-1} \partial_{+} g\right)^{i}=L_{+}^{(l)^{i}}=\partial_{+} x^{\mu}{ }_{\mu} L^{(l)^{i}}, \\
& \left(g^{-1} \partial_{-} g\right)^{j}=L_{-}^{(l)^{j}}={ }^{j} L_{\nu}^{(l)^{t}} \partial_{-} x^{\nu},
\end{aligned}
$$

where the superscript $t$ stands for supertransposition. On the other hand, by use of $<{ }_{i} v, L^{(l) j}>={ }_{i} \delta^{j}$ with $L^{(l) j}=\vec{d} x^{\nu}{ }_{\nu} L^{(l) j}$ we have

$$
{ }_{i} v^{\mu}={ }_{i} L^{(l) \mu} \mu^{-1} \quad, \quad(-1)^{i+i \nu \nu} L^{(l)-t}={ }_{i} v^{\nu} .
$$

\footnotetext{
${ }^{4}$ Here one must use of superdeterminant and superinverse formula [16].
} 
Then from (3.9) we will have

$$
\begin{aligned}
& A_{+i}(g)=(-1)^{i+i \nu} \partial_{+} x^{\mu}{ }_{\mu} \mathcal{E}_{\nu}{ }_{i} v^{\nu}, \\
& A_{-i}(g)=-(-1)^{i}{ }_{i} v^{\mu}{ }_{\mu} \mathcal{E}_{\nu} \partial_{-} x^{\nu}
\end{aligned}
$$

where

$$
{ }_{\mu} \mathcal{E}_{\nu}={ }_{\mu} L^{(l)^{i}}{ }_{i} E_{j}^{+}(g)^{j} L_{\nu}^{(l)^{t}}
$$

Now using the above relations in (3.10) we attain the proper result. Note that by use of

$$
\left(g^{-1} \partial_{ \pm} g\right)=R_{ \pm}^{(l)^{i}} g^{-1}{ }_{i} X g=R_{ \pm}^{(l)^{i}}{ }_{i} a^{(l)^{j}}(g)_{j} X
$$

where

$$
\left(\partial_{ \pm} g g^{-1}\right)^{i}=R_{ \pm}^{(l)^{i}}
$$

we have

$$
L_{ \pm}^{(l)^{i}}=R_{ \pm}^{(l)^{j}} a^{(l)^{i}}(g),
$$

and one can rewrite the action of (3.11) in the following form:

$$
S=\frac{1}{2} \int\left(\partial_{+} g g^{-1}\right)^{i}{ }_{i} \mathbb{E}_{j}^{+}(g)\left(\partial_{-} g g^{-1}\right)^{j} d \xi^{+} d \xi^{-},
$$

where

$$
{ }_{i} \mathbb{E}_{j}^{+}(g)={ }_{i}\left(E^{+^{-}}(e)+\Pi(g)\right)_{j}^{-1}
$$

and the super Poisson structure has the following form:

$$
\Pi=b a^{-1} \text {. }
$$

In the same way one can obtain the following super Poisson-Lie symmetric dual sigma model

$$
\tilde{S}=\frac{1}{2} \int\left(\partial_{+} \tilde{g} \tilde{g}^{-1}\right)_{i} \tilde{\mathbb{E}}^{i j}(\tilde{g})_{j}\left(\partial_{-} \tilde{g} \tilde{g}^{-1}\right) d \xi^{+} d \xi^{-}
$$

with

$$
\tilde{\mathbb{E}}^{i j}(\tilde{g})=\left(\tilde{E}^{+^{-}}(\tilde{e})+\tilde{\Pi}(\tilde{g})\right)^{i j^{-1}}
$$

where

$$
E^{ \pm}(e) \tilde{E}^{ \pm}(\tilde{e})=\tilde{E}^{ \pm}(\tilde{e}) E^{ \pm}(e)=I
$$

\section{Examples}

In this section we consider Poisson-Lie T-dual sigma models related to four dimensional Lie super bialgebras $\left(\left(2 A_{1,1}+2 A\right)^{1}, D_{p=\frac{1}{2}}^{10}\right)$ and $\left(\left(2 A_{1,1}+2 A\right)^{1}, I\right)^{5}$.

\footnotetext{
${ }^{5}$ These Lie super-bialgebras are obtained in $[19]$ in the same way as $[18$. Note that for the Lie superalgebras with odd numbers of fermionic coordinates the metric tensor is singular 16 , hence we consider four dimensional Lie superalgebras with two fermionic coordinates as an example.
} 


\subsection{Case A}

For Lie super-bialgebra $\left(\left(2 A_{1,1}+2 A\right)^{1}, D_{p=\frac{1}{2}}^{10}\right)$, we have the following nonzero (anti) commutation relations to the basis ${ }^{6}\left\{X_{1}, X_{2}, X_{3}, X_{4}\right\}$ for $\left(2 A_{1,1}+2 A\right)^{1}$ and $\left\{\tilde{X}^{1}, \tilde{X}^{2}, \tilde{X}^{3}, \tilde{X}^{4}\right\}$ for $D_{p=\frac{1}{2}}^{10}$

$$
\begin{aligned}
& \left\{X_{3}, X_{3}\right\}=X_{1}, \quad,\left\{X_{4}, X_{4}\right\}=X_{2}, \\
& {\left[\tilde{X}^{1}, \tilde{X}^{2}\right]=\tilde{X}^{2}, \quad\left[\tilde{X}^{1}, \tilde{X}^{3}\right]=\frac{3}{2} \tilde{X}^{3},} \\
& {\left[\tilde{X}^{1}, \tilde{X}^{4}\right]=\frac{1}{2} \tilde{X}^{4}, \quad\left[\tilde{X}^{2}, \tilde{X}^{4}\right]=\tilde{X}^{3},} \\
& {\left[X_{2}, \tilde{X}^{1}\right]=X_{2}, \quad\left[X_{2}, \tilde{X}^{2}\right]=-X_{1}, \quad\left[X_{3}, \tilde{X}^{1}\right]=\frac{3}{2} X_{3}-\tilde{X}^{3},} \\
& {\left[X_{3}, \tilde{X}^{2}\right]=X_{4}, \quad\left[X_{4}, \tilde{X}^{1}\right]=\frac{1}{2} X_{4}, \quad\left[X_{4}, \tilde{X}^{2}\right]=-\tilde{X}^{4},} \\
& \left\{X_{3}, \tilde{X}^{3}\right\}=\frac{3}{2} X_{1}, \quad\left\{X_{3}, \tilde{X}^{4}\right\}=X_{2}, \quad\left\{X_{4}, \tilde{X}^{4}\right\}=\frac{1}{2} X_{1} .
\end{aligned}
$$

Now using (3.17) with the following representation for the Lie supergroups $\left(\mathbf{2} \mathbf{A}_{\mathbf{1 , 1}}+\mathbf{2} \mathbf{A}\right)^{\mathbf{1}}$ and $\mathbf{D}_{\mathbf{P}=\frac{1}{2}}^{10}$ :

$$
g=e^{x X_{1}} e^{y X_{2}} e^{\psi X_{3}} e^{\chi X_{4}}, \quad \tilde{g}=e^{\tilde{x} \tilde{X}^{1}} e^{\tilde{y} \tilde{X}^{2}} e^{\tilde{\psi} \tilde{X}^{3}} e^{\tilde{\chi} \tilde{X}^{4}},
$$

where $\{x, y, \tilde{x}, \tilde{y}\}$ and $\{\psi, \chi, \tilde{\psi}, \tilde{\chi}\}$ are bosonic and fermionic coordinates of the Lie supergroups $\left(\mathbf{2} \mathbf{A}_{\mathbf{1}, \mathbf{1}}+\mathbf{2} \mathbf{A}\right)^{\mathbf{1}}$ and $\mathbf{D}_{\mathbf{P}=\frac{1}{2}}^{\mathbf{1 0}} ;$ we have

$$
\begin{gathered}
R_{ \pm}^{(l)^{i}}=\left(\begin{array}{cccc}
\partial_{ \pm} x-\frac{\psi}{2} \partial_{ \pm} \psi & \partial_{ \pm} y-\frac{\chi}{2} \partial_{ \pm} \chi & -\partial_{ \pm} \psi & -\partial_{ \pm} \chi
\end{array}\right), \\
\Pi^{i j}(g)=\left(\begin{array}{cccc}
0 & -y & \frac{3 \psi}{2} & \frac{\chi}{2} \\
y & 0 & 0 & \psi \\
-\frac{3 \psi}{2} & 0 & 0 & 0 \\
-\frac{\chi}{2} & -\psi & 0 & 0
\end{array}\right) .
\end{gathered}
$$

Then using (3.19) and choosing ${ }_{i} E_{j}^{+}(e)$ as

$$
{ }_{i} E_{j}^{+}(e)=\left(\begin{array}{cccc}
1 & 0 & 0 & 0 \\
0 & 1 & 0 & 0 \\
0 & 0 & 0 & 1 \\
0 & 0 & -1 & 0
\end{array}\right),
$$

the following model is derived

$$
\begin{aligned}
S & =\frac{1}{2} \int \frac{1}{2\left(1+y^{2}\right)}\left\{\left(2-\frac{3 \psi \chi}{1+y^{2}}\right)\left(\partial_{+} x \partial_{-} x+y \partial_{+} x \partial_{-} y-y \partial_{+} y \partial_{-} x\right)\right. \\
& +\left(2+\frac{3 y^{2} \psi \chi}{1+y^{2}}\right) \partial_{+} y \partial_{-} y-[\chi+(1+2 y) \psi] \partial_{+} x \partial_{-} \psi \\
& -[-\chi+(1+2 y) \psi] \partial_{+} \psi \partial_{-} x+(3 \psi-y \chi)\left(\partial_{+} x \partial_{-} \chi-\partial_{+} \chi \partial_{-} x\right) \\
& +[y \chi+(y-2) \psi] \partial_{+} y \partial_{-} \psi+[y \chi-(y-2) \psi] \partial_{+} \psi \partial_{-} y \\
& -(\chi+3 y \psi)\left(\partial_{+} y \partial_{-} \chi+\partial_{+} \chi \partial_{-} y\right)+\left[2\left(1+y^{2}\right)+(5-y) \frac{\psi \chi}{2}\right] \partial_{+} \psi \partial_{-} \chi
\end{aligned}
$$

\footnotetext{
${ }^{6}$ Here $\left\{X_{1}, X_{2}, \tilde{X}^{1}, \tilde{X}^{2}\right\}$ and $\left\{X_{3}, X_{4}, \tilde{X}^{3}, \tilde{X}^{4}\right\}$ are bosonic and fermionic bases respectively [22].
} 


$$
\left.-(1+2 y) \psi \chi \partial_{+} \psi \partial_{-} \psi-\left[2\left(1+y^{2}\right)+(1+y) \frac{\psi \chi}{2}\right] \partial_{+} \chi \partial_{-} \psi\right\} d \xi^{+} d \xi^{-},
$$

where the action have the following background matrices

$$
\begin{gathered}
{ }_{\mu} G_{\nu}=\frac{1}{2\left(1+y^{2}\right)}\left(\begin{array}{cccc}
2-\frac{3 \psi \chi}{1+y^{2}} & 0 & -\chi & 3 \psi-y \chi \\
0 & 2+\frac{3 y^{2} \psi \chi}{1+y^{2}} & (y-2) \psi & 0 \\
-\chi & (y-2) \psi & 0 & 2\left(1+y^{2}\right)+\frac{3}{2} \psi \chi \\
3 \psi-y \chi & 0 & -2\left(1+y^{2}\right)-\frac{3}{2} \psi \chi & 0
\end{array}\right), \\
{ }_{\mu} B_{\nu}=\frac{1}{2\left(1+y^{2}\right)}\left(\begin{array}{cccc}
0 & \left(2-\frac{3 \psi \chi}{1+y^{2}}\right) y & -(1+2 y) \psi & 0 \\
-\left(2-\frac{3 \psi \chi}{1+y^{2}}\right) y & 0 & y \chi & -(\chi+3 y \psi) \\
(1+2 y) \psi & -y \chi & -(1+2 y) \psi \chi & \frac{1}{2}(2-y) \psi \chi \\
0 & (\chi+3 y \psi) & \frac{1}{2}(2-y) \psi \chi & 0
\end{array}\right)
\end{gathered}
$$

For the dual model with

$$
\begin{aligned}
& \tilde{R}^{(l)}{ }_{i}=\left(\begin{array}{llll}
\partial_{ \pm} \tilde{x} & e^{\tilde{x}} \partial_{ \pm} \tilde{y} & e^{\frac{3 \tilde{x}}{2}}\left(\partial_{ \pm} \tilde{\psi}+\tilde{y} \partial_{ \pm} \tilde{\chi}\right) & e^{\frac{\tilde{x}}{2}} \partial_{ \pm} \tilde{\chi}
\end{array}\right), \\
& \tilde{\Pi}_{i j}(\tilde{g})=\left(\begin{array}{cccc}
0 & 0 & 0 & 0 \\
0 & 0 & 0 & 0 \\
0 & 0 & -\frac{1}{3}\left(\tilde{y}^{3} e^{3 \tilde{x}}+e^{3 \tilde{x}}-1\right) & -\frac{\tilde{y}^{2}}{2} e^{2 \tilde{x}} \\
0 & 0 & -\frac{\tilde{y}^{2}}{2} e^{2 \tilde{x}} & -\tilde{y} e^{\tilde{x}}
\end{array}\right),
\end{aligned}
$$

and

$$
\tilde{E}^{i j}(\tilde{e})=\left(\begin{array}{cccc}
1 & 0 & 0 & 0 \\
0 & 1 & 0 & 0 \\
0 & 0 & 0 & -1 \\
0 & 0 & 1 & 0
\end{array}\right)
$$

we have

$$
\begin{aligned}
\tilde{S} & =\frac{1}{2} \int\left\{\partial_{+} \tilde{x} \partial_{-} \tilde{x}+e^{2 \tilde{x}} \partial_{+} \tilde{y} \partial_{-} \tilde{y}-\frac{1}{\lambda}\left[\tilde{y} e^{4 \tilde{x}} \partial_{+} \tilde{\psi} \partial_{-} \tilde{\psi}\right.\right. \\
& +\left(\frac{\tilde{y}^{2}}{2} e^{4 \tilde{x}}-e^{2 \tilde{x}}\right) \partial_{+} \tilde{\psi} \partial_{-} \tilde{\chi}+\left(\frac{\tilde{y}^{2}}{2} e^{4 \tilde{x}}+e^{2 \tilde{x}}\right) \partial_{+} \tilde{\chi} \partial_{-} \tilde{\psi} \\
& \left.\left.+\frac{e^{\tilde{x}}}{3}\left(\tilde{y}^{3} e^{3 \tilde{x}}+e^{3 \tilde{x}}-3\right) \partial_{+} \tilde{\chi} \partial_{-} \tilde{\chi}\right]\right\} d \xi^{+} d \xi^{-},
\end{aligned}
$$

such that for the background matrices we have

$$
{ }_{\mu} \tilde{G}_{\nu}=\left(\begin{array}{cccc}
1 & 0 & 0 & 0 \\
0 & e^{2 \tilde{x}} & 0 & 0 \\
0 & 0 & 0 & \frac{e^{2 \tilde{x}}}{\lambda} \\
0 & 0 & -\frac{e^{2 \tilde{x}}}{\lambda} & 0
\end{array}\right), \quad{ }_{\mu} \tilde{B}_{\nu}=\left(\begin{array}{cccc}
0 & 0 & 0 & 0 \\
0 & 0 & 0 & 0 \\
0 & 0 & \frac{-\tilde{y} e^{4 \tilde{x}}}{\lambda^{\lambda}} & \frac{-\tilde{y}^{2} e^{4 \tilde{x}}}{2 \lambda} \\
0 & 0 & \frac{-\tilde{y}^{2} e^{4 \tilde{x}}}{2 \lambda} & \frac{-e^{\tilde{x}}}{3 \lambda}\left(\tilde{y}^{3} e^{3 \tilde{x}}+e^{3 \tilde{x}}-3\right)
\end{array}\right),
$$

where

$$
\lambda=\frac{\tilde{y}^{4}}{12} e^{4 \tilde{x}}+\frac{\tilde{y}}{3} e^{4 \tilde{x}}-\frac{\tilde{y}}{3} e^{\tilde{x}}+1
$$




\subsection{Case B}

For Lie super-bialgebra $\left(\left(2 A_{1,1}+2 A\right)^{1}, I\right)$, where $I$ is $(2,2)$ (with two bosonic and fermionic bases) Abelian superalgebra, we have the following nonzero (anti) commutation relations to the basis $\left\{X_{1}, X_{2}, X_{3}, X_{4}\right\}$ for $\left(2 A_{1,1}+2 A\right)^{1}$ and $\left\{\tilde{X}^{1}, \tilde{X}^{2}, \tilde{X}^{3}, \tilde{X}^{4}\right\}$ for $I$ :

$$
\begin{aligned}
& \left\{X_{3}, X_{3}\right\}=X_{1}, \quad\left\{X_{4}, X_{4}\right\}=X_{2}, \\
& {\left[X_{3}, \tilde{X}^{1}\right]=-\tilde{X}^{3}, \quad\left[X_{4}, \tilde{X}^{2}\right]=-\tilde{X}^{4} .}
\end{aligned}
$$

Using the representation in (4.2) we have $\Pi^{i j}(g)=0$ and if we choose ${ }_{i} E_{j}^{+}(e)$ as (4.4) the action of the model will have the following form

$$
\begin{aligned}
S & =\frac{1}{2} \int d \xi^{+} d \xi^{-}\left\{\partial_{+} x \partial_{-} x+\partial_{+} y \partial_{-} y+\partial_{+} \psi \partial_{-} \chi-\partial_{+} \chi \partial_{-} \psi\right. \\
& \left.-\frac{\psi}{2}\left(\partial_{+} x \partial_{-} \psi+\partial_{+} \psi \partial_{-} x\right)-\frac{\chi}{2}\left(\partial_{+} y \partial_{-} \chi+\partial_{+} \chi \partial_{-} y\right)\right\}
\end{aligned}
$$

with the background matrices as

$$
{ }_{\mu} G_{\nu}=\left(\begin{array}{cccc}
1 & 0 & 0 & 0 \\
0 & 1 & 0 & 0 \\
0 & 0 & 0 & 1 \\
0 & 0 & -1 & 0
\end{array}\right) \quad, \quad{ }_{\mu} B_{\nu}=\left(\begin{array}{cccc}
0 & 0 & -\frac{\psi}{2} & 0 \\
0 & 0 & 0 & -\frac{\chi}{2} \\
\frac{\psi}{2} & 0 & 0 & 0 \\
0 & \frac{\chi}{2} & 0 & 0
\end{array}\right)
$$

For the dual model we find

$$
\tilde{R}_{ \pm i}^{(l)}=\left(\begin{array}{llll}
\partial_{ \pm} \tilde{x} & \partial_{ \pm} \tilde{y} & \partial_{ \pm} \tilde{\psi} & \partial_{ \pm} \tilde{\chi}
\end{array}\right) \quad, \quad \tilde{\Pi}_{i j}(\tilde{g})=\left(\begin{array}{cccc}
0 & 0 & 0 & 0 \\
0 & 0 & 0 & 0 \\
0 & 0 & -\tilde{x} & 0 \\
0 & 0 & 0 & -\tilde{y}
\end{array}\right)
$$

then we have

$$
\begin{aligned}
\tilde{S} & =\frac{1}{2} \int d \xi^{+} d \xi^{-}\left\{\partial_{+} \tilde{x} \partial_{-} \tilde{x}+\partial_{+} \tilde{y} \partial_{-} \tilde{y}\right. \\
& \left.+\frac{1}{\tilde{x} \tilde{y}+1}\left(\partial_{+} \tilde{\psi} \partial_{-} \tilde{\chi}-\partial_{+} \tilde{\chi} \partial_{-} \tilde{\psi}-\tilde{y} \partial_{+} \tilde{\psi} \partial_{-} \tilde{\psi}-\tilde{x} \partial_{+} \tilde{\chi} \partial_{-} \tilde{\chi}\right)\right\}
\end{aligned}
$$

with the following background matrices

$$
{ }_{\mu} \tilde{G}_{\nu}=\left(\begin{array}{cccc}
1 & 0 & 0 & 0 \\
0 & 1 & 0 & 0 \\
0 & 0 & 0 & \frac{1}{\tilde{x} \tilde{y}+1} \\
0 & 0 & \frac{-1}{\tilde{x} \tilde{y}+1} & 0
\end{array}\right) \quad, \quad{ }_{\mu} \tilde{B}_{\nu}=\left(\begin{array}{cccc}
0 & 0 & 0 & 0 \\
0 & 0 & 0 & 0 \\
0 & 0 & \frac{-\tilde{y}}{\tilde{x} \tilde{y}+1} & 0 \\
0 & 0 & 0 & \frac{-\tilde{x}}{\tilde{x} \tilde{y}+1}
\end{array}\right) .
$$

Note that the background matrices of the model depend only on the fermionic fields $\{\psi, \chi\}$ and for the dual model the matrices depend only on the bosonic fields $\{\tilde{x}, \tilde{y}\}$. As such we see that in this case the super Poisson-Lie T-duality (super non-Abelian duality) transforms the role of fermionic fields in the model to bosonic fields on the dual model. In the next section we generalize this feature for general Abelian Lie super-bialgebra $(\mathbf{g}, \mathbf{I})$. But before 
that, let us investigate the physical equivalence of the model and its dual in the case B by use of canonical transformations. The generating functional for this equivalence is

$$
\begin{aligned}
F[x, \tilde{x}] & =-\frac{1}{2} \int d \sigma \tilde{x}_{i} R_{\sigma}^{\left(l^{i}\right.} \\
& =-\frac{1}{2} \int d \sigma\left\{\tilde{x} \partial_{\sigma} x-\tilde{x} \frac{\psi}{2} \partial_{\sigma} \psi+\tilde{y} \partial_{\sigma} y-\tilde{y} \frac{\chi}{2} \partial_{\sigma} \chi-\tilde{\psi} \partial_{\sigma} \psi-\tilde{\chi} \partial_{\sigma} \chi\right\},
\end{aligned}
$$

This generating functional produces the following canonical transformations

$$
p_{i}=\frac{\overleftarrow{\delta} F}{\delta x^{i}}, \quad \tilde{p}^{i}=-\frac{\overleftarrow{\delta} F}{\delta \tilde{x}_{i}}
$$

i.e.

$$
\begin{aligned}
p_{1} & =\frac{1}{2} \partial_{\sigma} \tilde{x}, & \tilde{p}^{1} & =\frac{1}{2} \partial_{\sigma} x-\frac{1}{4} \psi \partial_{\sigma} \psi, \\
p_{2} & =\frac{1}{2} \partial_{\sigma} \tilde{y}, & \tilde{p}^{2} & =\frac{1}{2} \partial_{\sigma} y-\frac{1}{4} \chi \partial_{\sigma} \chi, \\
p_{3} & =-\frac{1}{2} \tilde{x} \partial_{\sigma} \tilde{\psi}-\frac{1}{4} \psi \partial_{\sigma} \tilde{x}-\frac{1}{2} \partial_{\sigma} \tilde{\psi}, & \tilde{p}^{3} & =\frac{1}{2} \partial_{\sigma} \psi, \\
p_{4} & =-\frac{1}{2} \tilde{y} \partial_{\sigma} \tilde{\chi}-\frac{1}{4} \chi \partial_{\sigma} \tilde{y}-\frac{1}{2} \partial_{\sigma} \tilde{\chi}, & \tilde{p}^{4} & =\frac{1}{2} \partial_{\sigma} \chi,
\end{aligned}
$$

with these canonical transformations the Hamiltonian of the model

$$
\begin{aligned}
\mathcal{H} & =p_{1}^{2}+p_{2}^{2}+\psi p_{1} p_{4}+\frac{1}{2} \psi \chi p_{1} p_{2}+2 p_{3} p_{4}-\chi p_{2} p_{3}+\frac{1}{4} \partial_{\sigma} x \partial_{\sigma} x \\
& +\frac{1}{4} \partial_{\sigma} y \partial_{\sigma} y+\frac{1}{2} \partial_{\sigma} \psi \partial_{\sigma} \chi-\frac{1}{4} \psi \partial_{\sigma} x \partial_{\sigma} \psi-\frac{1}{4} \chi \partial_{\sigma} y \partial_{\sigma} \chi
\end{aligned}
$$

with

$$
p_{i}=\frac{\overleftarrow{\partial} L}{\partial\left(\partial_{\tau} x^{i}\right)}
$$

is equal to the Hamiltonian of the dual model

$$
\begin{aligned}
\tilde{\mathcal{H}}= & \left(\tilde{p}^{1}\right)^{2}+\left(\tilde{p}^{2}\right)^{2}+2(\tilde{x} \tilde{y}+1) \tilde{p}^{3} \tilde{p}^{4}+\tilde{x} \tilde{p}^{3} \partial_{\sigma} \tilde{\chi}-\tilde{y} \tilde{p}^{4} \partial_{\sigma} \tilde{\psi} \\
& +\frac{1}{2} \partial_{\sigma} \tilde{\psi} \partial_{\sigma} \tilde{\chi}+\frac{1}{4}\left(\partial_{\sigma} \tilde{x} \partial_{\sigma} \tilde{x}+\partial_{\sigma} \tilde{y} \partial_{\sigma} \tilde{y}\right) .
\end{aligned}
$$

with

$$
\tilde{p}^{i}=\frac{\overleftarrow{\partial} \tilde{L}}{\partial\left(\partial_{\tau} \tilde{x}_{i}\right)}
$$

Therefore the two models (4.13) and (4.16) are physically equivalent. 


\section{Super non-Abelian duality}

Here we consider Abelian Lie super-bialgebras $(\mathbf{g}, I)$ where $\mathbf{g}$ and $I$ have $(m, 2 n) \mathrm{m}$ bosonic and $2 n$ fermionic generators. We consider the following three cases in terms of commutation relations of Lie superalgebra $\mathbf{g}$ :

a) The commutation relations for the generators $\left\{X_{A}\right\}=\left\{X_{1}, \cdots, X_{m}, X_{m+1}, \cdots, X_{m+2 n}\right\}$ of $\mathbf{g}$ have the following form:

$$
\left[X_{i}, X_{m+a}\right]=\sum_{b=1}^{2 n} f_{i, m+a}^{m+b} X_{m+b}, \quad i=1, \cdots, m, \quad a=1, \cdots, 2 n,
$$

i.e. we have only commutation relation of bosons with fermions. Now by use of the following parameterizations for the Lie supergroup $\mathrm{G}$

$$
g=e^{x^{1} X_{1}} \cdots e^{x^{m} X_{m}} e^{\psi^{1} X_{m+1}} \cdots e^{\psi^{2 n} X_{m+2 n}},
$$

we have

$\partial_{ \pm} g g^{-1}=\sum_{i=1}^{m} \partial_{ \pm} x^{i} X_{i}+\sum_{a=1}^{2 n} \sum_{k_{1}, k_{2}, \cdots k_{m+a}=1}^{m+2 n} \partial_{ \pm} \psi^{a}\left(e^{-x^{m} \chi_{m}}\right)_{m+a}{ }^{k_{1}} \cdots\left(e^{-x^{1} \chi_{1}}\right)_{k_{m}}{ }^{k_{m+1}} X_{k_{m+a}}$

where $\chi_{m}$ is adjoint representation of the bosonic bases $X_{m}$. By comparison of this relation with (3.18) we see that the $R_{ \pm}^{(l)^{i}}$ are functions of bosonic coordinates of Lie supergroup $G$ i.e. $\left\{x^{i}\right\}$. On the other hand as for case $\mathrm{B}$ of section 4 we have

$$
b=\Pi=0, \quad E_{i j}^{+}(g)=E_{i j}^{+}(e) .
$$

In this way by use of (3.19) we see that the background matrix depends only on the bosonic coordinates of the Lie supergroup G. Furthermore by using of the commutation relations of Lie super-bialgebras for $(\mathrm{g}, I)$ we have [18]

$$
\begin{gathered}
{\left[X_{i}, \tilde{X}^{m+a}\right]=\sum_{b=1}^{2 n} f_{m+b, i}^{m+b} \tilde{X}^{m+b},} \\
{\left[X_{m+a}, \tilde{X}^{m+b}\right]=-\sum_{i=1}^{m} f_{i, m+a}^{m+b} \tilde{X}^{i},}
\end{gathered}
$$

then one can obtain

$$
\begin{gathered}
\tilde{g}^{-1} X_{i} \tilde{g}=X_{i}+\sum_{a=1}^{2 n} \sum_{b=1}^{2 n} \tilde{\psi}_{a} f_{m+b, i}^{m+a} \tilde{X}^{m+b}, \\
\tilde{g}^{-1} X_{m+a} \tilde{g}=X_{m+a}-\sum_{i=1}^{m} \sum_{b=1}^{2 n} \tilde{\psi}_{b} f_{m+a, i}^{m+b} \tilde{X}^{i},
\end{gathered}
$$

and by comparison of dual version of (3.8) we see that the matrix $\tilde{b}$ depends only on the fermionic coordinates of $\tilde{G}$; then $\tilde{\Pi}=\tilde{b} \tilde{a}^{-1}$ is only the function of fermionic coordinates 
of $\tilde{G}$ and background matrix of dual model depends only on the fermionic coordinates. In this case super Poisson Lie T-duality transforms the role of bosonic fields in the model to the fermionic fields on the dual model.

b) The commutation relations for the generators $\left\{X_{A}\right\}$ of $\mathbf{g}$ have the following form

$$
\left[X_{m+a}, X_{m+b}\right]=\sum_{i=1}^{m} f_{m+a, m+b}^{i} X_{i}
$$

i.e. we have only commutation relations of fermions with fermions. In this case we have

$$
\partial_{ \pm} g g^{-1}=\sum_{i=1}^{m} \partial_{ \pm} x^{i} X_{i}+\sum_{a=1}^{2 n} \partial_{ \pm} \psi^{a} X_{m+a}-\sum_{a>b=1}^{2 n} \sum_{i=1}^{m} \partial_{ \pm} \psi^{a} \psi^{b} f_{m+a, m+b}^{i} X_{i}
$$

then the background matrix of the model depends only on the fermionic fields. On the other hand, for the dual model we have

$$
\begin{gathered}
{\left[X_{m+a}, \tilde{X}^{i}\right]=-\sum_{b=1}^{2 n} f_{m+b, m+a}^{i} \tilde{X}^{m+b},} \\
\tilde{g}^{-1} X_{i} \tilde{g}=X_{i}, \quad \tilde{g}^{-1} X_{m+a} \tilde{g}=X_{m+a}+\sum_{i=1}^{m} \sum_{b=1}^{2 n} \tilde{x}_{i} f_{m+b, m+a}^{i} \tilde{X}^{m+b},
\end{gathered}
$$

then the matrix $\tilde{b}$ and background matrix of the dual model depend only on the bosonic fields. In this case super Poisson Lie T-duality transforms the role of fermionic fields in the model to the bosonic fields on the dual model (such as case B of section 4).

c) For the case

$$
\left[X_{i}, X_{j}\right]=\sum_{k=1}^{m} f_{i j}^{k} X_{k}
$$

we have only commutation relations of bosons with bosons; the background matrices of models and its dual are functions of bosonic fields and super Poisson Lie T-duality transforms the bosonic fields to the bosonics ones (such as ordinary Poisson Lie T-duality).

\section{Conclusion}

We investigated Poisson-Lie $T$-duality for sigma models on supermanifolds, especially on Lie supergroups. We show that for the Abelian case $(\mathrm{g}, I)$ the super Poisson Lie T-duality transforms the role of fermionic (bosonic) fields in the model to bosonic (fermionic) fields on the dual model and vice versa. We hope that the relationship between $T$-duality and mirror symmetry will be better understood in this way. Furthermore, one can investigate Poisson-Lie $T$-dual sigma models on supergroups having conformal symmetry, as well as its relations to superstring theories on $A d S$ backgrounds. The study of Poisson-Lie $T$-dual sigma models on low dimensional supergroups [18, 19] with spectator fields and its relations to models such as $2+1$ dimensional string cosmology coupled with fermionic matter can be considered an other open problems, some of which are under investigation. 


\section{Acknowledgments}

We would like to thank Sh. Moghadassi for carefully reading the manuscript and useful comments.

\section{References}

[1] V. Schomerus and H. Saleur, The GL(1|1) WZW model: From supergeometry to logarithmic CFT , Nucl. Phys. B 734 (2006) 221-245, hep-th/0510032.

[2] G.Gotz, T.Quella and V.Schomerus, The WZW model on PSU $(1,1 \mid 2)$, J. High Energy Phys. 0703 (2007) 003, hep-th/0610070.

[3] H. Saleur and V. Schomerus, On the $S U(2 \mid 1) W Z N W$ model and its statistical mechanics applications, Nucl. Phys. B 775 (2007) 312-340, hep-th/0611147.

[4] N.Berkovits, C.Vafa, E.Witten, Conformal Field Theory of Ads Backgrounds with Ramond-Ramond Flux, J. High Energy Phys. 9903 (1999) 018, hep-th/9902098.

[5] M. Bershadsky, S. Zhukov and A. Vaintrob, $P S L(n \mid n)$ sigma models as a conformal field theory, Nucl. Phys. B 559 (1999) 205-234, hep-th/9902180.

[6] N. Berkovits, M. Bershadsky, T. Hauer, S. Zhukov and B. Zwiebach, Superstring Theory on $A d S_{2} \times S_{2}$ as a Coset Supermanifold, Nucl. Phys. B 567 (2000) 61-86, hep-th/9907200.

[7] S. Sethi, Supermanifolds, Rigid Manifolds and Mirror Symmetry, Nucl. Phys. B 430 (1994) 31-50, hep-th/9404186.

[8] A. Schwarz, Sigma models having supermanifolds as target spaces, Lett. Math. Phys. 38 (1996) 91-96, hep-th/9506070.

[9] A. Giveon, M. Porrati, E.Rabinovici, Target Space Duality in String Theory, Phys. Rept. 244 (1994) 77-202, hep-th/9401139.

[10] C. Klimčik and P. Ševera, Dual non-Abelian duality and the Drinfeld double, Phys. Lett. B $351(1995)$ 445-462.

[11] C. Klimčik, Poisson-Lie T-duality, Nucl. Phys. 46 (Proc. Suppl.) (1996) 116-121, hep-th/9509095.

[12] C. Klimčik and P. Ševera, Poisson-Lie T-duality and loop groups Drinfeld doubles, Phys. Lett. B 372 (1996) 65-71, hep-th/9512040.

[13] M.A. Jafarizadeh and A. Rezaei-Aghdam, Poisson-Lie T-duality and Bianchi type algebras, Phys. Lett. B 458 (1999) 477-490, hep-th/9903152.

[14] V. G. Derinfeld, Quantum groups in: Proc.ICM. MSRI, Berkeley. 1986. p. 798.

[15] S. G. Parkhomenko, Mirror symmetry as a Poisson-Lie T-dulity, Mod. Phys. Lett. A 13 (1998) 1041-1054, hep-th/9710037.

[16] B. DeWitt, Supermanifold, Cambridge University Press 1992.

[17] N. Andruskiewitsch, Lie Superbialgebras and Poisson-Lie supergroups, Abh. Math. Sem. Univ. Hamburg 63 (1993). 147-163.

[18] A. Eghbali, F. Heidarpour and A. Rezaei-Aghdam, Classification of two and three dimensional Lie super-bialgebras, arXiv:0901.4471 [math-ph]. 
[19] A. Eghbali and A. Rezaei-Aghdam, Classification of four-dimensional Lie super-bialgebras of type $(2,2)$, work in progress.

[20] A. Eghbali and A. Rezaei-Aghdam, Classical r-matrices of two and three dimensional Lie super-bialgebras and their Poisson-Lie supergroups, arXiv:0908.2182 [math-ph].

[21] C. Juszczak and J. T. Sobczyk, Classification of low dimentional Lie super-bialgebras, J. Math. Phys. 39 (1998) 4982-4992.

[22] N. Backhouse, A classification of four-dimensional Lie superalgebras, J. Math. Phys. 19 (1978) 2400-2402. 